\title{
Neutralizing activity of BBIBP-CorV vaccine-elicited sera against multiple SARS-CoV-2 variants of concern
}

Xinxin Zhang ( $\square$ zhangx@shsmu.edu.cn )

Ruijin Hospital

\section{Xiaoqi Yu}

Ruijin Hospital

\section{Dong Wei}

Ruijin Hospital

\section{Wenxin Xu}

Ruijin Hospital

\section{Wentian Guo}

Ruijin Hospital

Xinxin Li

Ruijin Hospital

Wei Tan

Ruijin Hospital

\section{Leshan Liu}

Ruijin Hospital

Jieming Qu

Department of Pulmonary and Critical Care Medicine, Ruijin Hospital, Shanghai Jiao Tong University School of Medicine, Shanghai, China https://orcid.org/0000-0003-0464-544X

\section{Zhi-Tao Yang}

Ruijin Hospital, Shanghai Jiaotong University School of Medicine

\section{Erzhen Chen}

Ruijin Hospital

\section{Article}

Keywords: SARS-CoV-2, BBIBP-CorV, vaccine

Posted Date: May 25th, 2021

DOI: https://doi.org/10.21203/rs.3.rs-483022/v1 
License: (c) (i) This work is licensed under a Creative Commons Attribution 4.0 International License. Read Full License

Version of Record: A version of this preprint was published at Nature Communications on April 4th, 2022. See the published version at https://doi.org/10.1038/s41467-022-29477-0. 


\section{Abstract}

We assessed the safety and immunogenicity of an inactivated SARS-CoV-2 vaccine BBIBP-CorV, especially measured the resistance of four global variants of concern: Lineage B.1.1.7, Lineage B.1.351, Lineage P.1, and Lineage B.1.526 to neutralizing activity of vaccine-elicited sera. Among 1006 enrolled participants, no serious adverse event was reported within 28 days post-vaccination. Seroconversion of neutralizing antibodies was seen in 698 (91.84\%) of 760 healthcare workers, and the geometric mean titres (GMTs) of neutralizing antibody titre was 62.68 (57.02-68.91) after the second immunization. We found that 57 (12.13\%), 99 (20.97\%), and 114 (24.26\%) vaccine-elicited sera showed complete or partial loss of neutralizing activity against lineage B.1.1.7, lineage B.1.526, and lineage P.1, respectively, while 199 (42.34\%) vaccine-elicited sera preserved neutralizing activity against lineage B.1.351, albeit at relatively low dilutions. These data indicated that humoral responses against SARS-CoV-2 could be effectively induced in vaccine recipients, although diminished neutralization potency against multiple variants was observed.

\section{Introduction}

With the unprecedented morbidity of the COVID-19 pandemic, the efficacy of different vaccine candidates needs to be assessed across diverse populations. The absence of immunity in the population makes susceptible people vulnerable to further waves of SARS-CoV-2 infection, and healthcare workers are at particularly high risk. Sustained progress has been made in the development of the SARS-CoV-2 vaccine, and several vaccine candidates showed safety and immunogenicity against SARS-CoV-2 based on the clinical trials, including inactivated vaccines ${ }^{1-3}$, mRNA-based vaccines ${ }^{4,5}$, adenovirus-vectored vaccines ${ }^{6-}$ 8 , and recombinant protein subunit vaccines ${ }^{9}$. The inactivated vaccine candidate BBIBP-CorV developed by Sinopharm (Beijing, China) was safety and well-tolerated in healthy people, and can induce high levels of neutralizing antibody titres to provide protection against SARS-CoV- 2 according to their clinical data ${ }^{1}$. However, whether these vaccines have long-term protective efficacy is still under investigation.

Recently, the rapidly spreading SARS-CoV-2 variants including lineage B.1.1.7 detected in the United Kingdom, lineage B.1.351 detected in South Africa, lineage P.1 in Brazil, and B.1.526 in South America, were reported to be transmitted more efficiently and faster across the world. These variants contain multiple immune escape mutations in the receptor binding domain (RBD) of the spike glycoproteins, which are the targets of virus-neutralizing antibodies. Therefore, these newly identified variants of particular interest have raised great concern about the vaccine efficacy against these variants. To answer this question, a neutralization assay was performed using SARS-CoV-2-S pseudoviruses bearing either the Wuhan reference strain or the lineage B.1.1.7, lineage B.1.351, lineage P.1, and lineage B.1.526 spike protein. Here, we report the safety and immunogenicity of an inactivated SARS-CoV-2 vaccine BBIBP-CorV in healthcare workers, especially evaluate the effect of diverse SARS-CoV-2 spike variants on vaccineelicited neutralization. 


\section{Results}

\section{Study participants}

Between January 14 and March 10, a total of 1006 healthcare workers in Shanghai Ruijin Hospital were enrolled in this study. The baseline characteristics of the participants are shown in Table 1. Of them, 284 were male and 722 were female, the mean age was 35.89 years. $169(16.80 \%)$ participants have at least one underlying disease.

\section{Safety outcomes}

No serious adverse events were noted. 447 (44.43\%) of 1006 vaccine recipients had at least one adverse reaction after either vaccination. The common adverse reactions were reported more often after the administration of the second dose (Table 2). The overall incidence of adverse reactions was 308 (30.62\%) of 1006 vaccine recipients after the second dose, compared with 241 (23.96\%) after the first dose.

At least one local adverse reaction occurred after either vaccination in $258(25.65 \%)$ of 1006 vaccine recipients. The proportion of vaccine recipients reporting local adverse reactions increased after the second dose. Pain at the injection-site was the most frequent solicited local adverse reaction, which was reported by 231 (22.96\%) participants, and was reported more frequently among vaccine recipients after the second dose (160 [15.91\%]) than among vaccine recipients after the first dose (97 [9.64\%]). The additional injection site adverse reaction included redness (65 [6.46\%]), swelling (50 [4.97\%]), and rash $(15[1.49 \%])$.

At least one systematic adverse reaction was reported by 310 (30.82\%) of 1006 vaccine recipients after either vaccination, and the most common systematic adverse reaction was fatigue, which was reported in $206(20.48 \%)$ vaccine recipients. The additional systemic adverse reactions followed by were headache (101 [10.04\%]), diarrhea (34 [3.38\%]), nausea and vomiting (31 [3.08\%]), fever (27 [2.68\%]), mucocutaneous abnormality (22 [2.19\%]), myalgia and arthralgia (18 [1.79\%]).

Clinical laboratory values revealed few mild to moderate transient abnormalities. After the first dose vaccination, 39 vaccine recipients had decreased hemoglobin, 51 had increased white blood cell count, two had increased lymphocyte count, 14 had increased neutrophils count, 31 had increased alanine aminotransferase, 49 had increased aspartate aminotransferase, 14 had increased serum total bilirubin, 40 had increased blood urea nitrogen, and two had increased creatinine. After the second dose vaccination, 27 vaccine recipients had decreased hemoglobin, 46 had increased white blood cell count, four had increased lymphocyte count, 15 had increased neutrophils count, 30 had increased alanine aminotransferase, 35 had increased aspartate aminotransferase, two had increased serum total bilirubin, 27 had increased blood urea nitrogen, and four had increased creatinine. No instances were considered as clinically significant.

\section{Immunogenicity response}


Immunological analyses were done among individuals who had sufficient blood samples. On the day of the first dose, none of the participants had any detectable specific antibodies against SARS-CoV-2 or neutralizing antibodies to the pseudoviruses. Rapid antibody responses to SARS-CoV-2 were observed in $609(63.17 \%)$ of 964 individuals who were collected sufficient blood samples for immunological analyses on day 21 after the first dose (Figure 1), and the median antibody level was 5.32 (2.32-13.35).

An unbiased set of post-immunization sera of 760 individuals were tested for neutralizing antibodies by day 28 after the whole-course vaccination. The seroconversion rate of neutralizing antibodies was 698 (91.84\%) of 760 individuals, and the GMT was 62.68 (95\% Cl 57.02-68.91). The specific antibodies against SARS-CoV-2 were detected in 731 (96.18\%) of 760 vaccine recipients, and the median antibody level was 33.96 (12.56-82.04). Sex was not a factor that affect the induction of neutralizing antibody. Vaccine recipients with subsequent seroconversion of neutralizing antibodies had significant younger age than those who did not achieve seroconversion of neutralizing antibodies (36.74 [9.92] vs. 40.50 [8.95], $\mathrm{p}<0.010)$.

In 470 selected participants with positive neutralizing activity against the Wuhan reference strain (GMT 68.72, [95\% $\mathrm{Cl}$ 61.97-76.20]), neutralization assays were also performed against multiple SARS-CoV-2 variants. As shown in Figure 2, 57 (12.13\%) vaccine-elicited sera showed complete or partial loss of neutralizing activity against lineage B.1.1.7, and the GMTs against B.1.1.7 declined 2.2-fold to 31.17 (95\% $\mathrm{Cl} 27.71-35.07)$ compared to their titres against wild-type strains. 99 (20.97\%) and 114 (24.26\%) vaccineelicited sera showed complete or partial loss of neutralizing activity against lineage B.1.526 and lineage P.1, respectively. The GMTs against lineage P.1 declined 1.9-fold to 37.07 (95\% $\mathrm{Cl}$ 27.71-35.07), whereas a marked decrease by 3.8-fold was observed in GMTs against lineage B.1.526 (18.21 [95\% Cl 16.40-20.23]). Only 199 (42.34\%) vaccine-elicited sera preserved neutralizing activity against lineage B.1.351, with significantly reduced GMTs $(15.08,[95 \% \mathrm{Cl} 13.06-17.42])$ compared with their titres against wild-type strains.

We further analyzed the consistency of seroconversion of the neutralizing antibody among these variants. Only 14 vaccine-elicited sera did not induce neutralizing antibodies to any of the mutant strains. Among 199 vaccine-elicited sera that were seropositive against lineage B.1.351, only one had negative neutralizing activity against the rest three variants. We than analyzed the neutralizing activity against the three variants including B.1.1.7, B.1.526, and P.1 with less resistance, $285(60.64 \%)$ vaccine-elicited sera preserved neutralizing activity against all three variants, and $354(75.32 \%)$ vaccine-elicited sera preserved neutralizing activity against any two variants.

\section{Cytokine responses}

The dynamic changes of several key inflammatory cytokines, including interferon- $\gamma$ (IFN- $\gamma$ ), interleukin-10 (IL-10), IL-12p70, IL-13, IL-2, IL-6, IL-8, and tumor necrosis factor-a (TNF-a), were chosen to be tested in serum at different time points. The levels of certain cytokines showed notable changes from the first dose through 28 days after the second dose (Figure 3). On 21 days post the first dose and on 28 days post the second dose, significant increases were observed in the levels of IFN- $\gamma$, IL-10, and IL-13 when 
compared with their levels on the day of the first dose. The levels of IL-8 and TNF-a showed significant increases on 21 days post the first dose, and then had significant decreases on 28 days post the second dose.

The cytokine response was lower among participants who did not successfully induce neutralizing antibody against SARS-CoV-2, including the levels of IFN- $\gamma$ and TNF- $a$ at 28 days post the whole-course vaccination, and the level of IL-12p70 on the day of the first dose (Figure 4).

\section{Discussion}

In this study, the inactivated vaccine BBIBP-CorV was safe and well-tolerated in healthcare workers. All adverse reactions were mild or moderate, and were reported more frequently among vaccine recipients after the second dose. The most frequently reported local adverse reaction was pain at the injection site, and the most common systematic adverse reaction was fatigue. There were no clinically significant abnormal changes in laboratory measurements.

The primary immunogenic endpoint was the seroconversion of neutralizing antibodies at day 28 post the second vaccination. The traditional neutralization assay for the SARS-CoV-2 vaccines using the isolated live virus must be handled at biosafety level 3 facilities, while a pseudotype virus-based neutralization assay against SARS-CoV-2 was developed and can be handled in biosafety level 2 facilities $^{10}$. Previous reports have shown good concordance between pseudotype neutralization and SARS-CoV-2 neutralization assays ${ }^{11}$, and it was proved to be a more sensitive, reproducible and robust method ${ }^{12}$. Therefore, we chose a much safer pseudovirus-based neutralization assay to evaluate the neutralizing activity in this study.

It should be noted that $62(8.16 \%)$ participants did not successfully induce neutralizing antibodies against SARS-CoV-2, which is higher than the previous reports. Data indicated that factors such as age, sex, and the presence of a coexisting condition might not affect the efficacy of certain COVID-19 vaccine ${ }^{4}$, while in this study, younger age was significantly related to the seroconversion of neutralizing antibodies. Since healthcare workers tend to have less sleep and more chaotic circadian rhythmicity than other population, whether sleep pattern is another factor that can impact vaccine efficacy remain undetermined. Sleep might boost virus-specific adaptive immunity and promote a cytokine milieu supporting cellular response, and lack of sleep in the night after vaccination was found to reduce antibody response to hepatitis $A$ vaccination, hepatitis $B$ vaccination, and influenza vaccination ${ }^{13}$. It can be reasonably inferred that extending sleep duration in the night after vaccination might result in higher antibody response, and further studies are advocated to collect more information that associated with the neutralizing activity to provide more conclusive evidence.

The emergence of new SARS-CoV-2 variants has led to concern about the potential of these variants to circumvent immunity elicited by natural infection or vaccination. One of the highly transmissible variants of particular interest: B.1.1.7, first detected in the United Kingdom, has a series of mutations in its spike 
protein, including N501Y and 69-70 del. N501Y mutation was of great concern because it is located in the receptor binding domain of the spike protein, and the viruses containing this mutation bind more tightly to their cellular receptor, ACE2 ${ }^{14}$. Lineage B.1.351, first identified in South Africa, carries the immune escape-associated mutation E484K. Many highly neutralizing monoclonal antibodies, most convalescent sera and mRNA-induced immune sera showed reduced inhibitory activity against viruses containing E484K mutaton ${ }^{15}$. Lineage P.1 is another highly transmissible variant first detected in Brazil, and it also contains E484K mutation. In addition to the three global variants of interest mentioned above, the newly identified variant B.1.526 with mutation E484K and additional mutations in the spike gene was considered as a new variant of concern in South America.

Most current data of neutralizing activity against SARS-CoV-2 variants (mainly focused on lineage B.1.1.7 and lineage B.1.351) is based on the nucleoside-modified RNA vaccine-elicited sera. Previous reports indicated that the vaccine-immune sera largely preserved neutralizing titres against the B.1.1.7

variant $^{16,17}$. However, significant decreases in titres of neutralizing antibodies against the B.1.351 variant were observed ${ }^{18,19}$. A recent study revealed that lineage B.1.1.7 showed little resistance to neutralizing activity, while lineage B.1.526 and lineage P.1 showed more resistance to the neutralization than the wildtype strains, but the sample size is relatively small ${ }^{20}$. Here, we provided data of neutralizing activity against four different newly identified global variants of concern, based on BBIBP-CorV-elicited sera with larger sample size. Consistent with previous studies, we found that $12.13 \%$ vaccine-elicited sera showed complete or partial loss of neutralizing activity against lineage B.1.1.7. At the same time, $20.97 \%$ and $24.26 \%$ vaccine-elicited sera showed complete or partial loss of neutralizing activity against lineage B.1.526 and lineage P.1 with E484K mutation, respectively. Similarly, the vaccine-elicited sera were significantly less effective in neutralizing lineage B.1.351, but still $42.34 \%$ vaccine-elicited sera preserved neutralizing activity against lineage B.1.351. It is not surprising that the neutralizing seropositive rate of lineage B.1.1.7 is higher than that of the other three variants with immune escape mutation E484K, but why lineage B.1.351 showed more resistance than lineage P.1 and lineage B.1.526 is still unclear.

In conclusion, we found that the inactivated SARS-CoV-2 vaccine BBIBP-CorV was safe and well-tolerated at both two doses in the recruited healthcare workers, and rapid humoral responses were induced after the first dose vaccination. $91.84 \%$ of participants had seropositive neutralizing antibodies against SARSCoV-2 after the whole-course vaccination. Sera elicited by an inactivated vaccine BBIBP-CorV showed diminished neutralization potency against lineage B.1.1.7, lineage B.1.526 and lineage P.1, especially lineage B.1.351, and the protective efficacy of the inactivated vaccine against the emerging variants remains to be determined.

\section{Methods}

\section{Study design and participants}

From January 14 to March 10, healthcare workers in Shanghai Ruijin Hospital who are willing to receive two doses, 21 days apart of inactivated SARS-CoV-2 vaccine (BBIBP-CorV, Sinopharm) were recruited in 
this study. Eligible participants were aged 18-59 years, negative for serum specific antibodies against SARS-CoV-2 at the time of screening (V1). Blood samples were taken from participants for serology tests on the day of the first dose (V2), on day 21 after the first dose (V3), and on day 28 after the second dose (V4). The protocol and informed consent were approved by the Ethics Committee of Shanghai Ruijin Hospital in accordance with the Declaration of Helsinki and Good Clinical Practice. Written informed consent from all participants was obtained before screening.

\section{Safety assessments}

Solicited local and systemic reactions were prompted by and recorded in an electronic diary, unsolicited adverse events and serious adverse events were assessed from the receipt of each dose. Laboratory safety measurements including hemoglobin, white blood cell count, lymphocyte count, neutrophils count, platelets, alanine aminotransferase, aspartate aminotransferase, serum total bilirubin, serum albumin, creatinine, and blood urea nitrogen were tested within 28 days post each vaccination.

\section{Immunogenicity assessments}

The specific antibodies against SARS-CoV-2 were measured using a chemiluminescence kit manufactured by Wantai BioPharm (China), the antibody levels were expressed by the chemiluminescence signal according to the manufacturer's instructions.

\section{Pseudovirus based neutralization assay}

A pseudovirus-based neutralization assay was used to assess the neutralization geometric mean titres (GMTs) of vaccine-elicited sera. The $50 \%$ inhibitory concentration $\left(\mathrm{IC}_{50}\right)$ was defined as the serum dilution at which the relative light units (RLUs) were reduced by $50 \%$ compared with the virus control wells. Serial dilutions of heat-inactivated sera (six dilutions in a 4-fold step-wise manner) were incubated with 250 $\mathrm{TCID}_{50}$ SARS-CoV-2 pseudoviruses expressing the reporter gene luciferase for 1 hour before seeding HEK293T-ACE2 cells in 96-well plates (20,000 cells/well). Following $48 \mathrm{~h}$ of incubation in a $5 \% \mathrm{CO}_{2}$ environment at $37^{\circ} \mathrm{C}$, the supernatant was removed, and the reduction in luciferase gene expression was measured. The $\mathrm{IC}_{50}$ values were calculated by generating a three-parameter non-linear regression curve fit in GraphPad Prism 8.4.0. A neutralizing antibody potency $<1: 4$ was considered negative. SARS-CoV-2 pseudoviruses bearing the spike protein from either the original Wuhan reference strain (wild-type) or lineage B.1.1.7, lineage B.1.351, Lineage P.1, and Lineage B.1.526 tested in this study were manufactured by Vazyme Biotech Co., Ltd (China).

\section{Cytokine measurement}

Inflammatory cytokines including interferon- $\mathrm{Y}$ (IFN-ץ), interleukin-10 (IL-10), IL-12p70, IL-13, IL-2, IL-6, IL-8, and tumor necrosis factor-a (TNF-a) using an electro-chemiluminescent multiplex assay (Meso Scale Discovery) to explore the underlying immune responses.

\section{Outcomes}


The primary safety endpoint was any adverse reactions within 28 days after each dose of vaccination. The secondary safety endpoint was any clinical laboratory abnormalities within 28 days after each dose of vaccination. The primary immunogenic endpoint was the seroconversion rate of neutralizing antibodies at day 28 post the second vaccination, and the secondary immunogenic endpoints were the geometric mean titres (GMTs) of neutralizing antibodies against SARS-CoV-2 at day 28 post the second vaccination.

\section{Statistical analysis}

Continuous variables with normal distribution were presented as mean \pm standard deviation [SD], and continuous variables that were not normally distributed were presented as median (interquartile range [IQR]). Categorical variables were described as count (\%). The statistical method used for analysis of antibody titres is the geometric mean (GMTs) and the corresponding $95 \%$ confidence interval $(95 \% \mathrm{Cl})$. The values were compared by Student's $t$ test, the Wilcoxon signed-rank test, of the Mann-Whitney U test as appropriate. To assess the distribution in different groups, a chi-square test (c2) was applied. Graphs were plotted using GraphPad Prism 8.4.0. Statistical analyses were done using SPSS 24.0. A two-sided $P$ value of less than 0.050 was considered statistically significant. This study is registered with ClinicalTrials.gov, NCT04795414.

\section{Declarations}

\section{Contributors}

$X Z, J Q, Z Y$, and $E C$ had the idea for and designed the study. XY, WX, WG, and LL were responsible for collecting and summarizing the clinical data. $X Y, D W, X L$, and $W T$ were responsible for antibody detection and neutralizing assay. XY, DW, WG, and WX processed statistical data. $X Y$ and $X Z$ drafted the manuscript. All authors reviewed and approved the final version.

\section{Declaration of interests}

The authors declare no competing interests.

\section{Data sharing}

The data that support the findings of this study are available from the corresponding authors on reasonable request.

\section{Acknowledgments}


This study was funded by Shanghai Key Laboratory of Emergency Prevention, Diagnosis and Treatment of Respiratory Infectious Diseases (20dz2261100), and a grant from Science and Technology Commission Shanghai Municipality (No. 20JC1410200). The investigators express their gratitude to Prof. Guang Ning for his great support. We thank all the participants involved in this study.

\section{References}

1. Xia, S., et al. Safety and immunogenicity of an inactivated SARS-CoV-2 vaccine, BBIBP-CorV: a randomised, double-blind, placebo-controlled, phase 1/2 trial. Lancet Infect Dis 21, 39-51 (2021).

2. Zhang, Y., et al. Safety, tolerability, and immunogenicity of an inactivated SARS-CoV-2 vaccine in healthy adults aged 18-59 years: a randomised, double-blind, placebo-controlled, phase $1 / 2$ clinical trial. Lancet Infect Dis 21, 181-192 (2021).

3. Xia, S., et al. Effect of an Inactivated Vaccine Against SARS-CoV-2 on Safety and Immunogenicity Outcomes: Interim Analysis of 2 Randomized Clinical Trials. JAMA 324, 951-960 (2020).

4. Polack, F.P., et al. Safety and Efficacy of the BNT162b2 mRNA Covid-19 Vaccine. N Engl J Med 383, 2603-2615 (2020).

5. Baden, L.R., et al. Efficacy and Safety of the mRNA-1273 SARS-CoV-2 Vaccine. N Engl J Med 384, 403-416 (2021).

6. Voysey, M., et al. Safety and efficacy of the ChAdOx1 nCoV-19 vaccine (AZD1222) against SARSCoV-2: an interim analysis of four randomised controlled trials in Brazil, South Africa, and the UK. Lancet 397, 99-111 (2021).

7. Logunov, D.Y., et al. Safety and efficacy of an rAd26 and rAd5 vector-based heterologous prime-boost COVID-19 vaccine: an interim analysis of a randomised controlled phase 3 trial in Russia. Lancet 397, 671-681 (2021).

8. Zhu, F.C., et al. Immunogenicity and safety of a recombinant adenovirus type-5-vectored COVID-19 vaccine in healthy adults aged 18 years or older: a randomised, double-blind, placebo-controlled, phase 2 trial. Lancet 396, 479-488 (2020).

9. Yang, S., et al. Safety and immunogenicity of a recombinant tandem-repeat dimeric RBD-based protein subunit vaccine (ZF2001) against COVID-19 in adults: two randomised, double-blind, placebo-controlled, phase 1 and 2 trials. Lancet Infect Dis (2021).

10. Nie, J., et al. Quantification of SARS-CoV-2 neutralizing antibody by a pseudotyped virus-based assay. Nat Protoc 15, 3699-3715 (2020).

11. Case, J.B., et al. Neutralizing Antibody and Soluble ACE2 Inhibition of a Replication-Competent VSVSARS-CoV-2 and a Clinical Isolate of SARS-CoV-2. Cell Host Microbe 28, 475-485 e475 (2020).

12. Nie, J., et al. Establishment and validation of a pseudovirus neutralization assay for SARS-CoV-2. Emerg Microbes Infect 9, 680-686 (2020).

13. Benedict, C. \& Cedernaes, J. Could a good night's sleep improve COVID-19 vaccine efficacy? Lancet Respir Med(2021). 
14. Starr, T.N., et al. Deep Mutational Scanning of SARS-CoV-2 Receptor Binding Domain Reveals Constraints on Folding and ACE2 Binding. Cell 182, 1295-1310 e1220 (2020).

15. Chen, R.E., et al. Resistance of SARS-CoV-2 variants to neutralization by monoclonal and serumderived polyclonal antibodies. Nat Med (2021).

16. Muik, A., et al. Neutralization of SARS-CoV-2 lineage B.1.1.7 pseudovirus by BNT162b2 vaccineelicited human sera. Science 371, 1152-1153 (2021).

17. Edara, V.V., Hudson, W.H., Xie, X., Ahmed, R. \& Suthar, M.S. Neutralizing Antibodies Against SARSCoV-2 Variants After Infection and Vaccination. JAMA (2021).

18. Liu, Y., et al. Neutralizing Activity of BNT162b2-Elicited Serum - Preliminary Report. N Engl J Med (2021).

19. Wu, K., et al. Serum Neutralizing Activity Elicited by mRNA-1273 Vaccine - Preliminary Report. N Engl $J$ Med (2021).

20. Wang, G.L., et al. Susceptibility of Circulating SARS-CoV-2 Variants to Neutralization. N Eng/ J Med (2021).

\section{Tables}

Table 1. Baseline demographic characteristics for all participants.

\begin{tabular}{lc}
\hline & Total $(\mathrm{n}=1006)$ \\
\hline Age & $35.89(9.10)$ \\
Sex & \\
\hline Male & $284(28.23 \%)$ \\
\hline Female & $722(71.77 \%)$ \\
\hline Han nationality & $1006(100 \%)$ \\
\hline Underlying diseases & $169(16.80 \%)$ \\
\hline Diabetes & $12(1.19 \%)$ \\
\hline Hypertension & $74(7.36 \%)$ \\
\hline
\end{tabular}

Data are expressed as number (\%) or mean (SD).

Table 2. Adverse reactions after the first and second doses of vaccination. 


\begin{tabular}{llll}
\hline \multirow{2}{*}{ Total adverse reactions } & \multicolumn{1}{c}{ Either dose } & \multicolumn{1}{c}{ Dose 1} & \multicolumn{1}{c}{ Dose 2} \\
\cline { 2 - 4 } Any local symptoms & $447(44.43 \%)$ & $241(23.96 \%)$ & $308(30.62 \%)$ \\
Pain & $258(25.65 \%)$ & $117(11.63 \%)$ & $175(17.40 \%)$ \\
Redness & $231(22.96)$ & $97(9.64 \%)$ & $160(15.91 \%)$ \\
Swelling & $65(6.46 \%)$ & $56(5.57 \%)$ & $11(1.09 \%)$ \\
Rash & $50(4.97 \%)$ & $24(2.39 \%)$ & $160(15.91 \%)$ \\
Any systemic symptoms & $15(1.49 \%)$ & $12(1.19 \%)$ & $5(0.50 \%)$ \\
Fatigue & $310(30.82 \%)$ & $177(17.59 \%)$ & $188(18.69 \%)$ \\
Headache & $206(20.48 \%)$ & $88(8.75 \%)$ & $134(13.32 \%)$ \\
Fever & $101(10.04 \%)$ & $72(7.16 \%)$ & $45(4.47 \%)$ \\
Diarrhea & $27(2.68 \%)$ & $19(1.89 \%)$ & $11(1.09 \%)$ \\
Nausea and vomiting & $34(3.38 \%)$ & $21(2.09 \%)$ & $16(1.59 \%)$ \\
Mucocutaneous abnormality & $22(2.19 \%)$ & $15(1.49 \%)$ & $10(0.99 \%)$ \\
Myalgia and arthalgia & $18(1.79 \%)$ & $14(1.39 \%)$ & $5(0.50 \%)$ \\
\hline
\end{tabular}

Data are expressed as number (\%)

Figures 


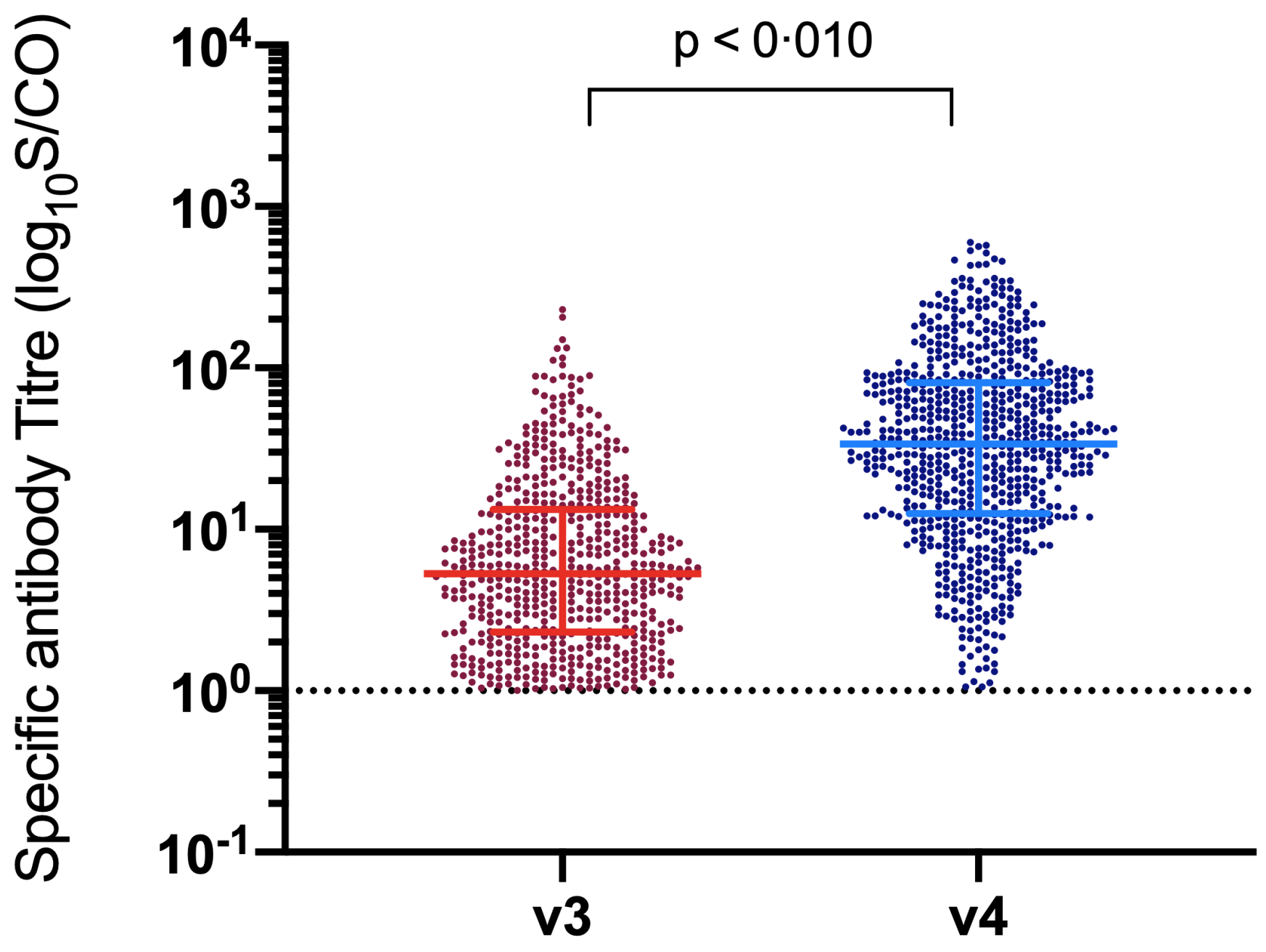

Figure 1

Antibody titres of serum specific antibodies against SARS-CoV-2. The spots indicated the individual antibody titres. The line in the scatter plot indicates the median and interquartile range (IQR). The horizontal dashed line represents the lower limit of detection of the assay (titre $<1$ ). Specific antibodies against SARS-CoV-2 were tested on day 21 after the first dose (V3) and on day 28 after the second dose (V4). $p$ value was calculated using the Mann-Whitney U test.
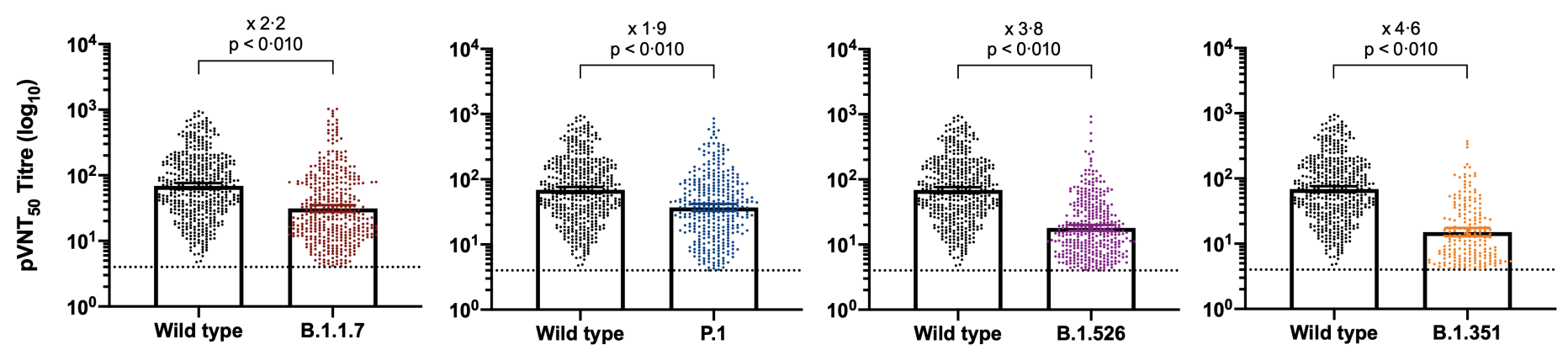

Figure 2 
Neutralization of variant strains of SARS-CoV-2 pseudoviruses after the second dose of vaccination. Shown are the results of 50\% pseudovirus neutralization titre (pVNT50) in 470 participants against Wildtype strain (Wuhan reference strain), lineage B.1.1.7 (United Kingdom), lineage B.1.351 (South Africa), lineage P.1 (Brazil), and lineage B.1.526 (South America). Box plots indicate the median and 95\% confidence interval $(95 \% \mathrm{Cl})$. Fold-changes in geometric mean titre are shown above the $\mathrm{p}$ value. The horizontal dashed line represents the lower limit of detection of the assay (titre $<4$ ). Analyses were performed with the use of Mann-Whitney $U$ test.

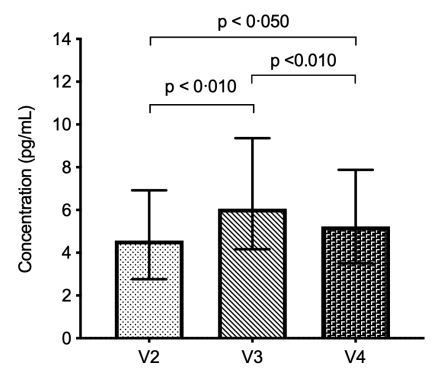

E

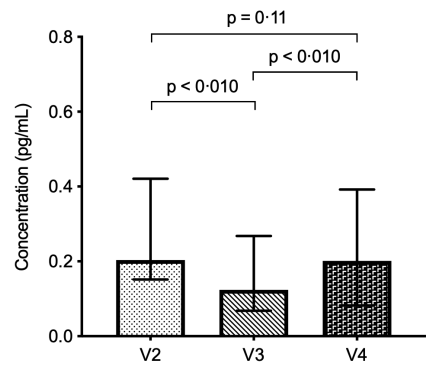

B

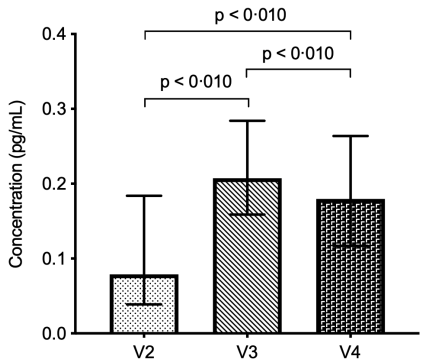

F

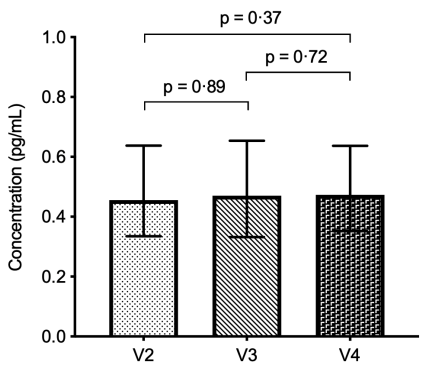

C

IL-12p70

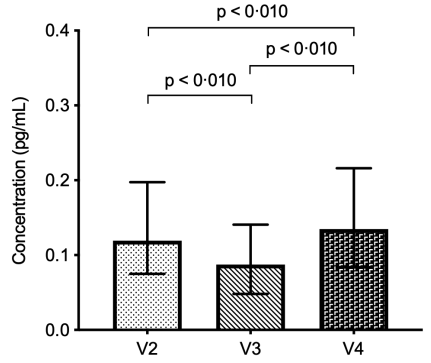

G

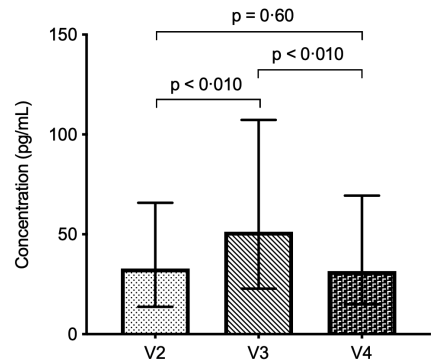

IL-13

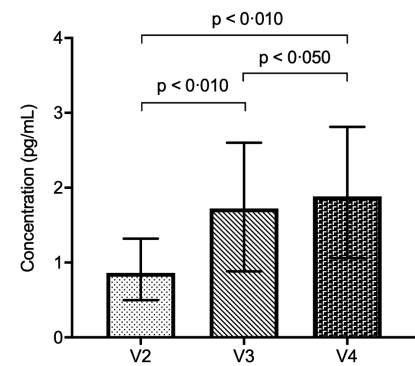

$\mathrm{H}$

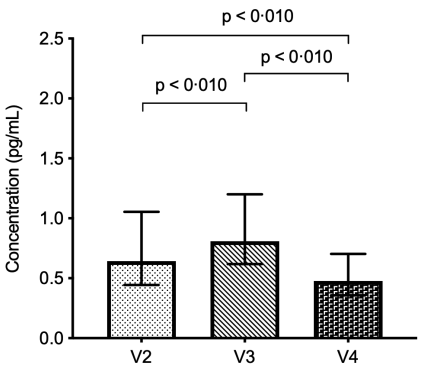

Figure 3

Dynamic changes in the levels of key inflammatory cytokines. The levels of key inflammatory cytokines including interferon- $\gamma$ (IFN- - ), interleukin-10 (IL-10), IL-12p70, IL-13, IL-2, IL-6, IL-8, and tumor necrosis factor- $a(T N F-a)$ were measured on the day of the first dose (V2), on day 21 after the first dose (V3), and on day 28 after the second dose (V4). Box plots indicate the median and interquartile range (IQR). $p$ values are shown on the figure. Analyses were performed with the use of Wilcoxon signed-rank test. 

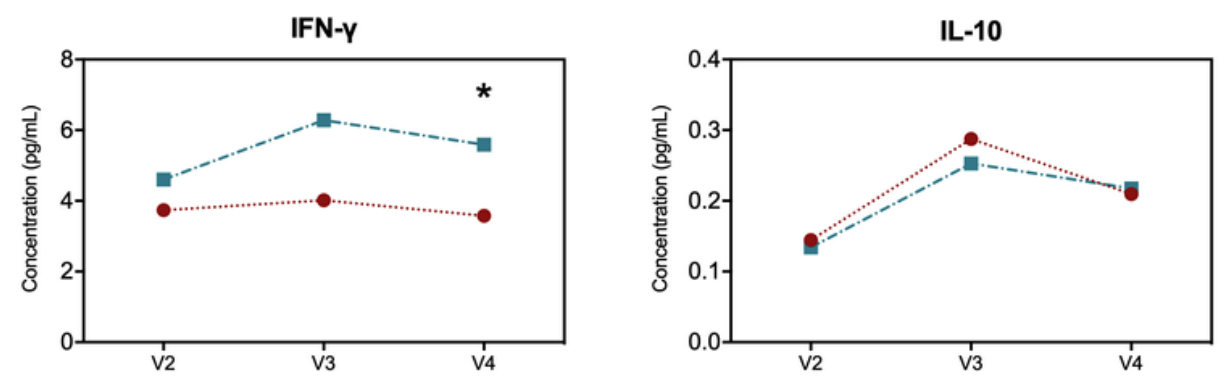

Negative

Positive
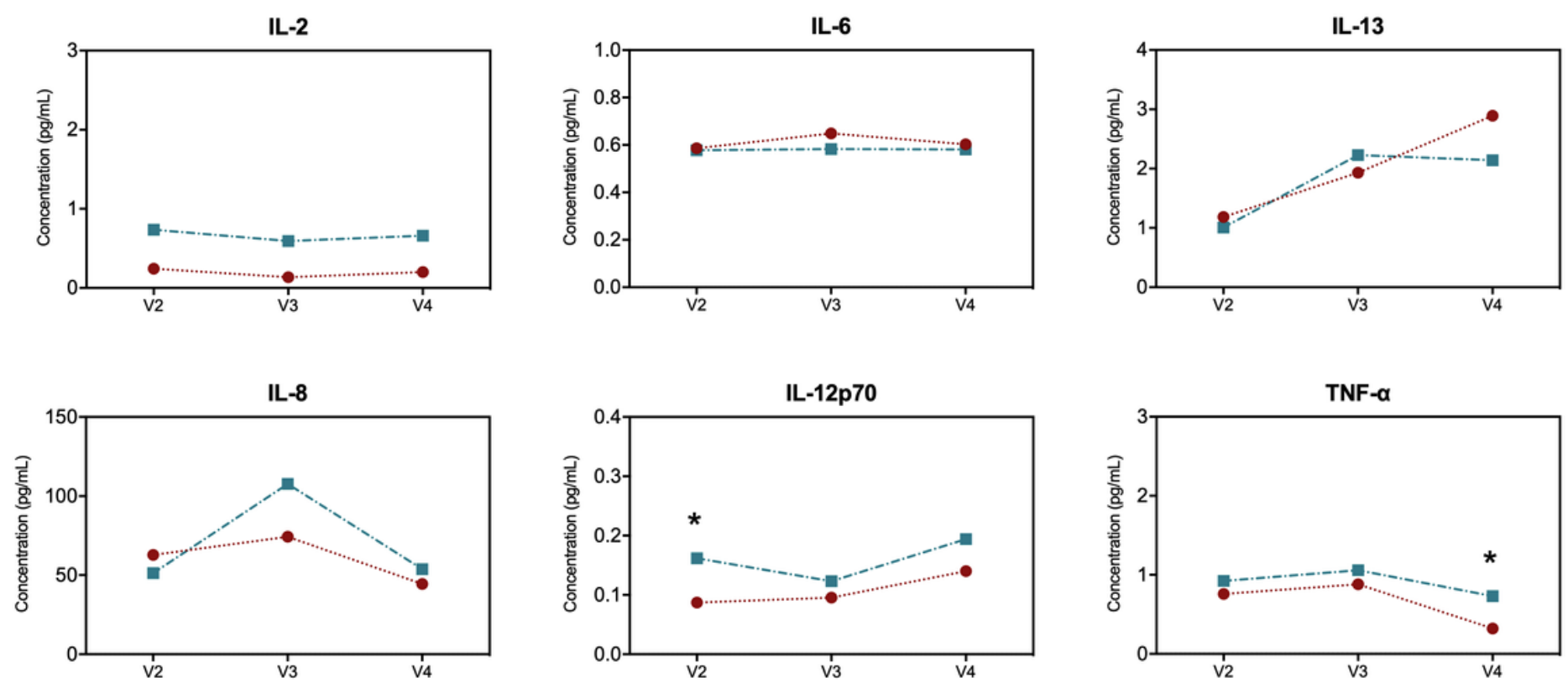

Figure 4

Sequential changes in the levels of key inflammatory cytokines in relation to neutralizing antibodies responses. The levels of key inflammatory cytokines between vaccine recipients with positive neutralizing antibodies and negative neutralizing antibodies. The dot indicated the median value. Only $p$ values of less than 0.050 (indicating significance) are shown. Negative, vaccine recipients with negative neutralizing antibodies. Positive, vaccine recipients with positive neutralizing antibodies.

\section{Supplementary Files}

This is a list of supplementary files associated with this preprint. Click to download.

- supplementaryappendixstudyprotocol.docx 